\title{
Correlation and Path Analysis for Yield and Yield Components in Blackgram [Vigna mungo (L.) Hepper]
}

\author{
Nikhil A. Suryawanshi*, Gabrial M. Lal and Suyog D. Warkad \\ Department of Genetics and Plant Breeding, Naini Agriculture Institute, Sam Higginbottom \\ University of Agriculture, Technology and Sciences, Allahabad-211007 (U.P.), India \\ *Corresponding author
}

\begin{tabular}{|l|}
\hline K e y w o r d s \\
Black gram [Vigna \\
mungo (L.) Hepper], \\
Genetic variability, \\
Correlation, Path analysis \\
\hline Article Info \\
\hline Accepted: \\
06 July 2018 \\
Available Online: \\
10 August 2018 \\
\hline
\end{tabular}

\section{A B S T R A C T}

The experimental material was consisting of 31 Black gram genotypes, check as T-9, during kharif 2017. The experiment was laid out in Randomised Complete Block Design with 3 replications at field experimentation centre of Department of Genetics and Plant Breeding, Sam Higginbottom University of Agriculture, Technology \& Sciences. The observations were logged on five randomly taken plants to each treatment and replication for 13 quantitative characters viz. days to $50 \%$ flowering, days to $50 \%$ pod setting, plant height, number of primary branches per plant, clusters per plant, pods per plant, pod length, seeds per pod, days to maturity, seed index, biological yield, harvest index and seed yield to estimate the variability, heritability and genetic advance as \% mean, character association and path analysis. High heritability coupled with high genetic advance as percent of mean was recorded for seed yield per plant represents simple selection is effective to improve these characters. The correlations revealed that harvest index and plant height have the significant positive association with the seed yield per plant at both genotypic and phenotypic levels. The path analysis revealed that the harvest index, biological yield, days to $50 \%$ flowering and seed per pod had shown the true relationship with seed yield by establishing the positive correlations and direct effects at both genotypic and phenotypic levels.

\section{Introduction}

Blackgram (Vigna mungo L. Hepper) is an important nutritious pulse crop occupying unique position in Indian agriculture. It belongs to family leguminoseae with chromosome number $2 n=2 x=22$. Blackgram is reported to be originated in India (Zukovskiji, 1962). India is the world's largest producer as well as consumer of blackgram. It produces about 1.5 to 1.9 million tons of blackgram annually from about 3.5 million hectares of area, with an average productivity of $500 \mathrm{~kg}$ per hectare. Blackgram output accounts for about $10 \%$ of India's total pulse production (Ministry of Agriculture, Govt. of India). In 2014-2015, 1.61 million tons Urad production in the country is largely concentrated in five states viz, Uttar Pradesh (UP), Maharashtra, Madhya Pradesh, Andhra Pradesh and Tamil Nadu. These five states together contribute for about $70 \%$ of total urad production in the country (Ministry of Agriculture, Govt of India). 
In U.P. Blackgram is grown in about 3.91 lakh hectares with a total production of 1.72 lakh tons (Annual Report 2014-2015). Among the states of India, Orissa ranks first in area 777 thousand hectares and production 396 thousand tones. However Punjab is a leading state in productivity with $834.9 \mathrm{~kg} /$ hectare (Kumar et al., 2002). It is a cheap source of dietary protein $(24 \%)$. It also contributes $76 \%$ carbohydrate, $3-5 \%$ Fibre, $1.74 \%$ Fat and a major portion of lysine in the vegetarian diet. It is the richest sources of phosphoric acid $\left(\mathrm{H}_{3} \mathrm{PO}_{4}\right)$. Being 5-10 times richer than other crops. Besides, being used as food for inexpensive source of dietary protein it is better to use for bean sprouts than mungbean for its longer shelf life (Mishra and Khan, 2001) Among pulses, blackgram (Vigna mungoL.) is an important short duration crop widely cultivated in India which give us an excellent source of easily digestible good quality protein and ability to restore the fertility of soil through symbiotic nitrogen fixation.In India black gram is grown both in winter and summer as monocrop and inter crop, respectively. That is why no single plant type is appropriate for all production system. So the variability among the existing germplasm or the accessions is the primary need to develop appropriate plant type for specific production system. Black gram originated in India where it has been in cultivation from ancient times and is one of the most highly prized pulses of India.

A successful breeding programme in blackgram would need information on the nature and degree of genetic divergence in the available stock for choosing the right parents for further improvement (Falconer, 1981).

\section{Materials and Methods}

The present investigation was carried out at the Field Experimentation Centre, Department of Genetics and Plant Breeding, Naini
Agricultural Institute, Sam Higginbottom University of Agriculture, Technology and Sciences, Allahabad, U.P. (India) during Kharif-2017.The experimental materials constituted of the germplasm collection of 31 genotypes of Black gram (Vigna mungo (L) Hepper), procured from Oilseed Research Station Jalgaon, Mahatma Phule Krishi Vidyapeeth, Rahuri. (Maharashtra) and Department of Genetics and Plant Breeding, SHUATS Allahabad (UP). Data were recorded from five randomly selected plants from each genotype per replication and the average was taken for analysis. All the recommended package of practices was followed to raise a good crop. The experiment was laid out in Randomised Complete Block Design with 3 replications. The genotypes were sown by hand dibbling in each plot by imposing randomisation in each replication along with check T-9. Each plot has 4 rows with the spacing of row to row $30 \mathrm{~cm}$ and plant to plant $10 \mathrm{~cm}$. Standard statistical procedures were used for the analysis of correlation coefficient values (r) at genotypic and phenotypic levels by Johnson et al., (1955a) and described by Singh and Choudhary (1985). Path coefficient analysis was utilized to partition the phenotypic and genotypic correlation coefficient into the direct effects and indirect effects along with residual effects. The analysis was carried out as per the equation suggested by Dewey and Lu (1959) originally proposed by Wright (1921) and described by Singh and Choudhary (1985).

\section{Results and Discussion}

The analysis of variance revealed highly significant to significant differences among the genotypes for all the thirteen characters studied (Table 1). In the present study, variation among the characters is estimated by Genotypic Coefficient of Variation (GCV) and Phenotypic Coefficient of Variation (PCV). The PCV was higher than the GCV for few 
characters indicates the interaction of genotypes with the environment (Table 2). Maximum PCV and GCV were recorded for Cluster per plant (31.39 and 22.99) followed by pod per plant (25.34 and 15.33), harvest index (21.34 and 15.10), seed yield per plant (16.43 and 14.86) and branches per plant (16.19 and 10.04). The estimates of heritability (\%) in the broad sense for 13 characters studied, which range from 20.93\% to $81.79 \%$. Maximum heritability (broad sense) was recorded for characters i.e., seed yield per plant $(81.79 \%)$, followed by seed index $(62.65 \%)$, days to maturity $(62.20 \%)$, clusters per plant $(53.29 \%)$, harvest index (50.09\%), days to $50 \%$ flowering $(48.53 \%)$, pod length (46.68\%), branches per plant (38.48\%), pods per plant $(36.56 \%)$.

Table.1 Analysis of variance for different characters of Black gram

\begin{tabular}{|l|l|c|c|c|}
\hline S. No. & Parameters & \multicolumn{3}{|c|}{ Mean Sum of Squares } \\
\hline $\mathbf{1}$ & Days to 50\% Flowering & $\begin{array}{c}\text { Replications } \\
\mathbf{( d f = 2}\end{array}$ & $\begin{array}{c}\text { Genotypes } \\
(\mathbf{d f}=\mathbf{4 0})\end{array}$ & $\begin{array}{c}\text { Error } \\
(\mathbf{d f}=\mathbf{8 0})\end{array}$ \\
\hline $\mathbf{2}$ & Days to 50\% Pod Setting & 0.1545 & $40.9000^{* *}$ & 0.5957 \\
\hline $\mathbf{3}$ & Plant Height (cm) & 0.1729 & $102.4780^{* *}$ & 1.9295 \\
\hline $\mathbf{4}$ & Branches/ Plant & 0.4315 & $0.2271^{* *}$ & 0.1469 \\
\hline $\mathbf{5}$ & Clusters/ Plant & 0.0315 & $5.7850^{* *}$ & 0.4205 \\
\hline $\mathbf{6}$ & Pods/ Plant & 0.0452 & $33.2665^{* *}$ & 0.7215 \\
\hline $\mathbf{7}$ & Pod Length (cm) & 0.2518 & $0.3487 * *$ & 0.0820 \\
\hline $\mathbf{8}$ & Seeds/ Pod & 0.0296 & $0.7776^{* *}$ & 0.3023 \\
\hline $\mathbf{9}$ & Days to Maturity & 2.2520 & $27.8175^{* *}$ & 1.3187 \\
\hline $\mathbf{1 0}$ & Seed Index (g) & 0.1642 & $1.2499^{* *}$ & 0.0600 \\
\hline $\mathbf{1 1}$ & Seed Yield/ Plant (g) & 0.1876 & $8.1714^{* *}$ & 0.3517 \\
\hline $\mathbf{1 2}$ & Biological Yield (g) & 1.7044 & $191.2677 * *$ & 1.4959 \\
\hline $\mathbf{1 3}$ & Harvest Index (\%) & 0.2681 & $32.8512^{* * *}$ & 1.4083 \\
\hline
\end{tabular}




\section{Int.J.Curr.Microbiol.App.Sci (2018) 7(8): 612-621}

Table.2 Genetic parameter of different characters in Black gram

\begin{tabular}{|c|c|c|c|c|c|c|c|c|}
\hline S.NO & Character & VG & $\mathbf{V P}$ & $\begin{array}{c}\text { GCV } \\
(\%)\end{array}$ & $\begin{array}{r}\text { PCV } \\
(\%)\end{array}$ & $\begin{array}{l}\mathbf{h}^{2} \mathbf{b s} \\
\%\end{array}$ & GA & $\underset{\text { mean }}{\text { GA as }}$ \% \\
\hline 1 & Days to $50 \%$ Flowering & 2.36 & 4.86 & 3.52 & 5.05 & 48.53 & 2.20 & 5.05 \\
\hline 2 & Days to $50 \%$ Pod Setting & 1.87 & 5.80 & 2.62 & 4.61 & 32.17 & 1.60 & 3.06 \\
\hline 3 & Plant Height $(\mathrm{cm})$ & 13.94 & 38.17 & 9.69 & 16.03 & 36.52 & 4.65 & 12.06 \\
\hline 4 & Branches/ Plant & 0.02 & 0.06 & 10.04 & 16.19 & 38.48 & 0.19 & 12.84 \\
\hline 5 & Clusters/ Plant & 4.31 & 8.09 & 22.99 & 31.49 & 53.29 & 3.12 & 34.57 \\
\hline 6 & Pods/ Plant & 27.85 & 76.18 & 15.33 & 25.34 & 36.56 & 6.57 & 19.09 \\
\hline 7 & Pod Length $(\mathrm{cm})$ & 0.03 & 0.06 & 4.06 & 5.94 & 46.68 & 0.24 & 5.71 \\
\hline 8 & Seeds/ Pod & 0.08 & 0.37 & 4.48 & 9.80 & 20.93 & 0.26 & 4.23 \\
\hline 9 & Days to Maturity & 1.48 & 2.38 & 1.38 & 1.75 & 62.20 & 1.98 & 2.24 \\
\hline 10 & Seed Index (g) & 0.25 & 0.40 & 9.59 & 12.12 & 62.65 & 0.82 & 15.64 \\
\hline 11 & Seed Yield/ Plant (g) & 1.72 & 2.10 & 14.86 & 16.43 & 81.79 & 2.44 & 27.69 \\
\hline 12 & Biological Yield (g) & 12.51 & 52.64 & 6.32 & 12.96 & 23.76 & 3.55 & 6.34 \\
\hline 13 & Harvest Index (\%) & 5.93 & 11.83 & 15.10 & 21.34 & 50.09 & 3.55 & 22.02 \\
\hline
\end{tabular}


Table.3 Correlation coefficient between yield and its related traits in 31 Black gram genotypes at Genotypic level

\begin{tabular}{|c|c|c|c|c|c|c|c|c|c|c|c|c|c|c|}
\hline $\begin{array}{l}\mathbf{N} \\
\mathbf{0}\end{array}$ & Character & $\begin{array}{l}\text { Days to } \\
50 \% \\
\text { Flowerin } \\
\text { g }\end{array}$ & $\begin{array}{l}\text { Days to } \\
50 \% \text { Pod } \\
\text { Setting }\end{array}$ & $\begin{array}{l}\text { Plant } \\
\text { Height }\end{array}$ & $\begin{array}{l}\text { Branche } \\
\text { s Per } \\
\text { Plant }\end{array}$ & $\begin{array}{l}\text { Clusters } \\
\text { Per } \\
\text { Plant }\end{array}$ & $\begin{array}{l}\text { Pods/ } \\
\text { Plant }\end{array}$ & $\begin{array}{l}\text { Pod } \\
\text { Length }\end{array}$ & $\begin{array}{l}\text { Seeds/ } \\
\text { Pod }\end{array}$ & $\begin{array}{l}\text { Days to } \\
\text { Maturity }\end{array}$ & $\begin{array}{l}\text { Seed } \\
\text { Index }\end{array}$ & $\begin{array}{l}\text { Biologica } \\
\text { I Yield }\end{array}$ & $\begin{array}{l}\text { harvest } \\
\text { Index }\end{array}$ & $\begin{array}{l}\text { Seed } \\
\text { Yield/ } \\
\text { Plant }\end{array}$ \\
\hline 1 & $\begin{array}{l}\text { Days to } 50 \% \\
\text { Flowering }\end{array}$ & 1.000 & $0.874 * *$ & $-0.661 * *$ & 0.054 & 0.068 & 0.037 & 0.092 & -0.124 & -0.083 & -0.079 & 0.060 & $-0.266 * *$ & $-0.241 *$ \\
\hline 2 & $\begin{array}{l}\text { Days to } 50 \% \text { Pod } \\
\text { Setting }\end{array}$ & & 1.000 & $-0.582 * *$ & -0.039 & -0.043 & -0.003 & -0.018 & $-0.397 * *$ & -0.003 & -0.117 & $0.265^{* *}$ & -0.110 & 0.017 \\
\hline 3 & Plant Height & & & 1.000 & -0.126 & $-0.520 * *$ & $-0.442 * *$ & $0.244^{*}$ & 0.097 & -0.043 & $0.470 * *$ & -0.086 & $0.610 * *$ & $0.562 * *$ \\
\hline 4 & Branches Per Plant & & & & 1.000 & $0.592 * *$ & $0.851 * *$ & 0.094 & $0.394 * *$ & 0.130 & -0.094 & 0.013 & -0.048 & 0.036 \\
\hline 5 & Clusters Per Plant & & & & & 1.000 & $0.908 * *$ & 0.010 & $0.394 * *$ & -0.127 & -0.182 & $0.600 * *$ & $-0.541 * *$ & $-0.297 * *$ \\
\hline 6 & Pods/ Plant & & & & & & 1.000 & 0.190 & $0.695 * *$ & -0.175 & -0.112 & $0.510 * *$ & $-0.471 * *$ & $0.262 *$ \\
\hline 7 & Pod Length & & & & & & & 1.000 & $1.054 * *$ & -0.196 & $0.555^{* *}$ & $-0.236^{*}$ & 0.155 & 0.021 \\
\hline 8 & Seeds/ Pod & & & & & & & & 1.000 & $-0.603 * *$ & $0.639 * *$ & $-0.218^{*}$ & $0.334 * *$ & 0.140 \\
\hline 9 & Days to Maturity & & & & & & & & & 1.000 & -0.091 & -0.115 & $-0.254 *$ & $-0.296 *$ \\
\hline 10 & Seed Index & & & & & & & & & & 1.000 & $-0.379 * *$ & 0.135 & 0.036 \\
\hline 11 & Biological Yield & & & & & & & & & & & 1.000 & $-0.349 * *$ & 0.016 \\
\hline 12 & harvest Index & & & & & & & & & & & & 1.000 & $0.939 * *$ \\
\hline 13 & Seed Yield/ Plant & & & & & & & & & & & & & 1.000 \\
\hline
\end{tabular}

$* \& * *$ Significant at $1 \%$ and $5 \%$ level of significance respectively 
Table.4 Correlation coefficient between yield and its related traits in 31 black gram genotypes at phenotypic level

\begin{tabular}{|c|c|c|c|c|c|c|c|c|c|c|c|c|c|c|}
\hline No & Character & $\begin{array}{l}\text { Days to } \\
50 \% \\
\text { Flowering }\end{array}$ & $\begin{array}{l}\text { Days to } \\
50 \% \\
\text { Pod } \\
\text { Setting }\end{array}$ & $\begin{array}{l}\text { Plant } \\
\text { Height }\end{array}$ & $\begin{array}{l}\text { Branches } \\
\text { Per Plant }\end{array}$ & $\begin{array}{l}\text { Clusters } \\
\text { Per } \\
\text { Plant }\end{array}$ & $\begin{array}{l}\text { Pods/ } \\
\text { Plant }\end{array}$ & $\begin{array}{l}\text { Pod } \\
\text { Length }\end{array}$ & $\begin{array}{l}\text { Seeds/ } \\
\text { Pod }\end{array}$ & $\begin{array}{l}\text { Days to } \\
\text { Maturity }\end{array}$ & $\begin{array}{l}\text { Seed } \\
\text { Index }\end{array}$ & $\begin{array}{l}\text { Biological } \\
\text { Yield }\end{array}$ & $\begin{array}{l}\text { harvest } \\
\text { Index }\end{array}$ & $\begin{array}{l}\text { Seed } \\
\text { Yield/ } \\
\text { Plant }\end{array}$ \\
\hline 1 & Days to $50 \%$ Flowering & 1.000 & $0.607 * *$ & $0.274 * *$ & -0.110 & 0.055 & 0.001 & -0.057 & -0.071 & -0.029 & -0.163 & 0.057 & -0.143 & -0.116 \\
\hline 2 & $\begin{array}{l}\text { Days to } 50 \% \text { Pod } \\
\text { Setting }\end{array}$ & & 1.000 & -0.135 & -0.093 & 0.014 & -0.012 & -0.096 & -0.110 & 0.019 & -0.123 & 0.076 & -0.088 & -0.039 \\
\hline 3 & Plant Height & & & 1.000 & 0.079 & -0.185 & -0.131 & $0.206^{*}$ & 0.090 & -0.057 & $0.277 * *$ & -0.127 & 0.313 & $0.285^{* *}$ \\
\hline 4 & Branches Per Plant & & & & 1.000 & $0.292 * *$ & $0.303 * *$ & 0.192 & 0.190 & 0.036 & 0.060 & 0.018 & -0.013 & 0.023 \\
\hline 5 & Clusters Per Plant & & & & & 1.000 & $0.841 * *$ & 0.069 & $0.249 *$ & -0.123 & -0.122 & 0.130 & -0.186 & -0.147 \\
\hline 6 & Pods/ Plant & & & & & & 1.000 & $0.235^{*}$ & $0.346^{* *}$ & -0.047 & -0.033 & 0.075 & -0.102 & 0.084 \\
\hline 7 & Pod Length & & & & & & & 1.000 & $0.568 * *$ & -0.078 & $0.355 * *$ & 0.022 & -0.036 & -0.036 \\
\hline 8 & Seeds/ Pod & & & & & & & & 1.000 & $-0.261 *$ & 0.145 & 0.087 & -0.067 & -0.011 \\
\hline 9 & Days to Maturity & & & & & & & & & 1.000 & 0.048 & -0.079 & -0.105 & $-0.202^{*}$ \\
\hline 10 & Seed Index & & & & & & & & & & 1.000 & $-0.241 *$ & 0.145 & 0.011 \\
\hline 11 & Biological Yield & & & & & & & & & & & 1.000 & - & 0.019 \\
\hline 12 & harvest Index & & & & & & & & & & & & 1.000 & $0.760 * *$ \\
\hline 13 & Seed Yield/ Plant & & & & & & & & & & & & & 1.000 \\
\hline
\end{tabular}

*\&** Significant at $1 \%$ and $5 \%$ level of significance respectively 
Table.5 Direct and indirect effects between yield and its related traits in 31 Black gram genotypes at genotypic level

\begin{tabular}{|c|c|c|c|c|c|c|c|c|c|c|c|c|c|c|}
\hline No & Character & $\begin{array}{r}\text { Days to } \\
50 \% \\
\text { Flowering }\end{array}$ & $\begin{array}{r}\text { Days to } \\
50 \% \text { Pod } \\
\text { Setting }\end{array}$ & $\begin{array}{r}\text { Plant } \\
\text { Height }\end{array}$ & $\begin{array}{l}\text { Branches } \\
\text { Per Plant }\end{array}$ & $\begin{array}{l}\text { Clusters } \\
\text { Per Plant }\end{array}$ & $\begin{array}{l}\text { Pods/ } \\
\text { Plant }\end{array}$ & $\begin{array}{r}\text { Pod } \\
\text { Length }\end{array}$ & $\begin{array}{r}\text { Seeds/ } \\
\text { Pod }\end{array}$ & $\begin{array}{l}\text { Days to } \\
\text { Maturity }\end{array}$ & $\begin{array}{l}\text { Seed } \\
\text { Index }\end{array}$ & $\begin{array}{r}\text { Biological } \\
\text { Yield }\end{array}$ & $\begin{array}{r}\text { harvest } \\
\text { Index }\end{array}$ & $\begin{array}{l}\text { Seed } \\
\text { Yield/ } \\
\text { Plant }\end{array}$ \\
\hline 1 & Days to $50 \%$ Flowering & 0.7369 & 0.6441 & -0.4876 & 0.0404 & 0.0503 & 0.0273 & 0.0678 & -0.0918 & -0.0618 & -0.0587 & 0.0443 & -0.1967 & -0.2412 \\
\hline 2 & Days to $50 \%$ Pod Setting & -0.7073 & -0.8093 & 0.4715 & 0.0315 & 0.0355 & 0.0029 & 0.0153 & 0.3214 & 0.0031 & 0.0947 & -0.2145 & 0.0892 & 0.0175 \\
\hline 3 & Plant Height & 0.3039 & 0.2676 & -0.4593 & 0.0583 & 0.2390 & 0.2031 & -0.1125 & -0.0448 & 0.0198 & -0.2159 & 0.0396 & -0.2802 & 0.5625 \\
\hline 4 & Branches Per Plant & -0.0158 & 0.0112 & 0.0366 & -0.2881 & -0.1708 & -0.2453 & -0.0273 & -0.1136 & -0.0377 & 0.0273 & -0.0037 & 0.0139 & 0.0367 \\
\hline 5 & Clusters Per Plant & -0.0645 & 0.0414 & 0.4912 & -0.5595 & -0.9440 & -0.8572 & -0.0095 & -0.3725 & 0.1207 & 0.1724 & -0.5671 & 0.5116 & -0.2975 \\
\hline 6 & Pods/ Plant & 0.0235 & -0.0023 & -0.2800 & 0.5390 & 0.5749 & 0.6331 & 0.1208 & 0.4402 & -0.1110 & -0.0710 & 0.3232 & -0.2986 & 0.2623 \\
\hline 7 & Pod Length & -0.0599 & 0.0123 & -0.1595 & -0.0616 & -0.0065 & -0.1242 & -0.6512 & -0.6866 & 0.1281 & -0.3614 & 0.1538 & -0.1012 & 0.0213 \\
\hline 8 & Seeds/ Pod & -0.0694 & -0.2213 & 0.0543 & 0.2197 & 0.2198 & 0.3874 & 0.5874 & 0.5571 & -0.3364 & 0.3565 & -0.1218 & 0.1864 & 0.1408 \\
\hline 9 & Days to Maturity & -0.0408 & -0.0019 & -0.0210 & 0.0638 & -0.0623 & -0.0853 & -0.0958 & -0.2940 & 0.4869 & -0.0443 & -0.0562 & -0.1239 & -0.2960 \\
\hline 10 & Seed Index & -0.0195 & -0.0286 & 0.1152 & -0.0232 & -0.0447 & -0.0275 & 0.1359 & 0.1567 & -0.0223 & 0.2449 & -0.0930 & 0.0333 & 0.0369 \\
\hline 11 & Biological Yield & 0.0599 & 0.2646 & -0.0862 & 0.0130 & 0.5996 & 0.5094 & -0.2357 & -0.2181 & -0.1153 & -0.3789 & 0.9980 & -0.3490 & 0.0062 \\
\hline 12 & harvest Index & -0.3882 & -0.1604 & 0.8874 & -0.0699 & -0.7883 & -0.6861 & 0.2260 & 0.4867 & -0.3703 & 0.1975 & -0.5088 & 1.4547 & 0.9394 \\
\hline
\end{tabular}

Bold are direct effects, R SQUARE $=94.68$, RESIDUAL EFFECT $=23.07$. 
Table.6 Direct and indirect effects between yield and its related traits in 31 Blackgram genotypes at phenotypic level

\begin{tabular}{|c|c|c|c|c|c|c|c|c|c|c|c|c|c|c|}
\hline No & Character & $\begin{array}{r}\text { Days to } \\
50 \% \\
\text { Flowering }\end{array}$ & $\begin{array}{r}\text { Days to } \\
\mathbf{5 0 \%} \\
\text { Pod } \\
\text { Setting }\end{array}$ & $\begin{array}{r}\text { Plant } \\
\text { Height }\end{array}$ & $\begin{array}{l}\text { Branches } \\
\text { Per Plant }\end{array}$ & $\begin{array}{r}\text { Clusters } \\
\text { Per } \\
\text { Plant }\end{array}$ & $\begin{array}{l}\text { Pods/ } \\
\text { Plant }\end{array}$ & $\begin{array}{r}\text { Pod } \\
\text { Length }\end{array}$ & $\begin{array}{r}\text { Seeds/ } \\
\text { Pod }\end{array}$ & $\begin{array}{r}\text { Days to } \\
\text { Maturity }\end{array}$ & $\begin{array}{r}\text { Seed } \\
\text { Index }\end{array}$ & $\begin{array}{r}\text { Biological } \\
\text { Yield }\end{array}$ & $\begin{array}{r}\text { harvest } \\
\text { Index }\end{array}$ & $\begin{array}{r}\text { Seed } \\
\text { Yield/ } \\
\text { Plant }\end{array}$ \\
\hline 1 & Days to $50 \%$ Flowering & 0.0182 & 0.0111 & -0.0050 & -0.0020 & 0.0010 & 0.0000 & -0.0010 & -0.0013 & -0.0005 & -0.0030 & 0.0010 & -0.0026 & -0.1156 \\
\hline 2 & Days to $50 \%$ Pod Setting & 0.0004 & 0.0007 & -0.0001 & -0.0001 & 0.0000 & 0.0000 & -0.0001 & -0.0001 & 0.0000 & -0.0001 & 0.0001 & -0.0001 & -0.0388 \\
\hline 3 & Plant Height & 0.0033 & 0.0016 & -0.0119 & -0.0009 & 0.0022 & 0.0016 & -0.0025 & -0.0011 & 0.0007 & -0.0033 & 0.0015 & -0.0037 & 0.2851 \\
\hline 4 & Branches Per Plant & 0.0016 & 0.0013 & -0.0011 & -0.0143 & -0.0042 & -0.0043 & -0.0027 & -0.0027 & -0.0005 & -0.0009 & -0.0003 & 0.0002 & 0.0233 \\
\hline 5 & Clusters Per Plant & -0.0004 & -0.0001 & 0.0014 & -0.0022 & -0.0076 & -0.0064 & -0.0005 & -0.0019 & 0.0009 & 0.0009 & -0.0010 & 0.0014 & -0.1468 \\
\hline 6 & Pods/ Plant & 0.0000 & 0.0001 & 0.0011 & -0.0026 & -0.0073 & -0.0087 & -0.0021 & -0.0030 & 0.0004 & 0.0003 & -0.0007 & 0.0009 & 0.0844 \\
\hline 7 & Pod Length & 0.0007 & 0.0011 & -0.0024 & -0.0022 & -0.0008 & -0.0027 & -0.0115 & -0.0065 & 0.0009 & -0.0041 & -0.0002 & 0.0004 & -0.0358 \\
\hline 8 & Seeds/ Pod & -0.0014 & -0.0021 & 0.0017 & 0.0036 & 0.0048 & 0.0066 & 0.0109 & 0.0191 & -0.0050 & 0.0028 & 0.0017 & -0.0013 & -0.0113 \\
\hline 9 & Days to Maturity & 0.0000 & 0.0000 & 0.0001 & 0.0000 & 0.0002 & 0.0001 & 0.0001 & 0.0003 & -0.0012 & -0.0001 & 0.0001 & 0.0001 & -0.2021 \\
\hline 10 & Seed Index & -0.0009 & -0.0007 & 0.0015 & 0.0003 & -0.0007 & -0.0002 & 0.0019 & 0.0008 & 0.0003 & 0.0055 & -0.0013 & 0.0008 & 0.0106 \\
\hline 11 & Biological Yield & 0.0463 & 0.0615 & -0.1024 & 0.0144 & 0.1051 & 0.0604 & 0.0176 & 0.0707 & -0.0636 & -0.1947 & 0.8087 & -0.5215 & 0.0194 \\
\hline 12 & harvest Index & -0.1834 & -0.1133 & 0.4021 & -0.0172 & -0.2394 & -0.1308 & -0.0459 & -0.0856 & -0.1344 & 0.1859 & -0.8290 & 1.2855 & 0.7602 \\
\hline
\end{tabular}

Bold are direct effects, R SQUARE $=95.85$, Residual Effect $=20.36$. 
The genotypic and phenotypic correlation coefficients were computed among 13 characters (Table 3). The genotypic correlations revealed that harvest index, pods per plant and plant height have the significant positive association with the seed yield per plant at genotypic levels, while days to $50 \%$ flowering, cluster per plant, days to maturity showing negative but significant association with seed yield at genotypic level.

Therefore, these characters appeared as greatest important associates of seed yield per plant and have also been observed by preceding workers (Kumar et al., 2005; Kumar et al., 2008; Sharma et al., 2005; Issacs et al., 2000; Katna and Verma, 2001).

The phenotypic correlations revealed that harvest index and plant height have the significant positive association with the seed yield per plant at phenotypic levels, while only days to maturity showing negative but significant association with seed yield at genotypic level.

Path coefficient analysis is a statistical technique to split the observed correlation coefficients into direct and indirect effects of independent variables on the dependent variable. In the present study, path coefficient analysis was carried out using genotypic and phenotypic correlation matrix of 13 characters (Table 4). The path analysis revealed that the harvest index, biological yield, seed index, seeds per pod, days to $50 \%$ flowering had shown the true relationship with seed yield by establishing the positive correlations and direct effects at both genotypic and phenotypic levels (Table 5 and 6). By considering the nature and extent of correlation coefficients and their direct and indirect effects it can be concluded that improvement of Black gram seed yield is brought through simultaneous selection seeds per pod, pod per plant, biological yield and harvest index.

\section{References}

Bind, B., Kumar, R., Bhind, H. N. and Sharma, V. (2014). Correlation and path analysis for yield and yield components in black gram (Vigna mungo (L.) Hepper). International Journal of Plant Sciences, 9 (2):410-413.

Falconer, D. S. (1981). Introduction to quantitative genetics, $3^{\text {rd }}$ ed. Longman, New York.: 340.

Idress, A., Sadiq, M. S., Hanif, M., Abbas, G. and Haider, S. (2006). Genetic parameters and path coefficient analysis in mutated generation of mungbean (Vigna radiate (L.) Wilczek) Agriculture. Research, 44 (3):22-26.

Konda, C. R., Salimath, P. M., and Mishra, M. N. (2009). Genetic variability studies for productivity and its components in blackgram (Vigna mungo (L.) Hepper). Legume Research, 32 (1):59-61.

Kumar, G. V., Vanaja, M., Sathish, P., Vagheera, P., and Lakhsmi, J. N. (2015). Correlation analysis for quantitative traits in blackgram (Vigna mungo (L.) Hepper) in different seasons.International Journal of Scientific and Research Publications, 5 (4):1-4.

Kumar, R., Singh, A., Rath, A. S., kumar, R. and Singh, A. (2002). Estimating genetic parameters in urd bean. Annals of Agriculture Research, 21: (3)335-337.

Mishra, D. K. and Khan, R. A. (2001). Stability analysis in urdbean for grain yield and associated traits.Indian Journal of Pulses Research, 14: (2)145-147.

Parveen, S. L, Sekhar, M. R., Reddy, D. M. and Sudhakar, P. (2011). Correlation and path coefficient analysis for yield and yield components in blackgram (Vigna mungo (1.) hepper). International Journal of Applied Biology and Pharmaceutical Technology, 2 (3):619625. 
Reni, Y. P., Rao Y. K., Satish Y. and Babu, J. S. (2013). Estimates of genetic parameters and path analysis in blackgram (Vigna mungo (1.) Hepper). International Journal of Plant, Animal and Environmental Sciences, 3 (4): 231234.

Shivade, H.A., A.P. Rewale and S.B. Patil (2011). Correlationand path analysis for yield and yield components in blackgram (Vigna mungo (L.) Hepper). Legume Res, 34 (3): $178-183$.

Srivastava, R. L. and Singh, G. (2012) Genetic variability, correlation and path analysis in mungbean (Vigna radiata (1.) wilczek). Indian Journal of Life Science, 2 (1):61-65.

Usharani, K. S. and kumar, A. C. R. (2015). Ems induced mutations in urdbean (Vigna mungo 1.) and significance of induced altered correlations. Life Sciences Leaflets, 63:140-146.

Zukovskiji, P. M. (1962). Cultivated plants and their wild relative (Abridged Transition by P. S. Hadsos: Commonwealth Agricultural Bureaux, Farham, Royals, Bucks).

\section{How to cite this article:}

Nikhil A. Suryawanshi, Gabrial M. Lal and Suyog D. Warkad. 2018. Correlation and Path Analysis for Yield and Yield Components in Blackgram [Vigna mungo (L.) Hepper]. Int.J.Curr.Microbiol.App.Sci. 7(08): 612-621. doi: https://doi.org/10.20546/ijcmas.2018.708.066 